\title{
Rastall's theory of gravity: Spherically symmetric solutions and the stability problem
}

\author{
K. A. Bronnikov ${ }^{1}$ \\ Center fo Gravitation and Fundamental Metrology, VNIIMS, Ozyornaya ul. 46, Moscow 119361, Russia; \\ Institute of Gravitation and Cosmology, RUDN University, ul. Miklukho-Maklaya 6, Moscow 117198, \\ Russia; \\ National Research Nuclear University "MEPhI", Kashirskoe sh. 31, Moscow 115409, Russia
}

\author{
Júlio C. Fabris ${ }^{2}$ \\ Núcleo Cosmo-ufes 8 Departamento de Física, CCE, Universidade Federal do Espírito Santo, \\ Vitória, ES, CEP29075-910, Brazil \\ National Research Nuclear University "MEPhI", Kashirskoe sh. 31, Moscow 115409, Russia
}

\begin{abstract}
Oliver F. Piattella
Núcleo Cosmo-ufes 85 Departamento de Física, CCE, Universidade Federal do Espírito Santo, Vitória, ES, CEP29075-910, Brazil
\end{abstract}

\section{Denis C. Rodrigues}

PPGFis, CCE, Universidade Federal do Espírito Santo, Vitória, ES, CEP29075-910, Brazil

\section{Edison C.O. Santos}

PPGCosmo, CCE, Universidade Federal do Espírito Santo, Vitória, ES, CEP29075-910, Brazil

\begin{abstract}
We study the stability of static, spherically symmetric solutions of Rastall's theory in the presence of a scalar field with respect to spherically symmetric perturbations. It is shown that the stability analysis is inconsistent in the sense that linear time-dependent perturbations cannot exist, and we can conclude that these solutions are stable. Possible reasons for this inconsistency are discussed.
\end{abstract}

\section{Introduction}

One of the fundamental pillars of the General Relativity (GR) theory of gravity is the divergencefree property of the Einstein tensor, leading to the usual conservation laws for the matter sources of gravity, expressed in the zero divergence of the energy-momentum tensor.

Rastall's theory [1] is a non-Lagrangian theory of gravity which gives up the above property of GR and allows for an energy-momentum tensor of matter which has a nonzero divergence. The

\footnotetext{
${ }^{1}$ e-mail: kb20@yandex.ru

${ }^{2}$ e-mail: julio.fabris@cosmo-ufes.org
} 
main original argument for such a radical departure from GR is that, strictly speaking, the conservation laws have only be tested in flat space, and their generalization to curved space-time may be more problematic than is usually supposed. The departure from the usual GR conservation law is parametrized, in the Rastall theory by the dimensionless parameter $\lambda$, such that when $\lambda=1$, GR is recovered.

The Rastall theory has been applied in many different contexts. In cosmology, some studies have been performed for the early [2] and also later universe [3. In some of these applications, a self-interacting scalar field has been considered. A curiosity about the self-interacting scalar field in the Rastall theory is that the corresponding speed of sound $c_{s}^{2}$ can be zero for some value of $\lambda$ [4], unlike the GR situation where always $c_{s}^{2}=1$ (in unities of the velocity of light). Moreover, It is possible to construct a cosmological model entirely similar to the standard $\Lambda$ CDM model for the background and linear perturbations, but different for nonlinear perturbations.

In Ref. [5] black-hole solutions using Rastall's theory in the presence of a scalar field have been obtained. Two classes of exotic black holes have been identified: one where the singularities are located at two spatial infinities separated by horizons; the other where there are two horizons of infinite area connected by a wormhole, and the spatial infinity is essentially formed by a cosmological singularity. It is very interesting that these structures are geometrically identical to those found using k-essence theories, but with a different behavior of the scalar fields [6]. In Ref. [7] the equivalence (or duality) between these structures was discussed in detail: we have formulated the assumptions under which the two fundamentally different theories lead to space-times with the same metric.

A question that emerges immediately concerns the stability of these exotic black hole-type structures. In Ref. [8] the stability of the corresponding k-essence solutions under spherically symmetric perturbations was analyzed, and their instabilities have been proved. In the present paper, we undertake a similar analysis for the Rastall black hole-type solutions. A surprising aspect of this study is that the linear perturbation analysis turns out to be inconsistent, and the stability or instability of the solutions cannot be determined. More precisely, the linear perturbation equations cannot have time-dependent solutions. Such a result looks similar to the cosmological perturbation analysis for a self-interacting scalar field, which has also revealed an inconsistency, unless new degrees of freedom of matter are added to the previously existing matter content of the Universe [9]. This may point out at an intrinsic restriction for the Rastall theory and perhaps even for a large class of non-Lagrangian theories of gravity.

\section{Basic equations}

The field equations of Rastall's theory where the only source of gravity is a scalar field $\phi$ with an arbitrary self-interaction potential $V(\phi)$ can be written in the form 3

$$
\begin{aligned}
& R_{\mu \nu}-\frac{1}{2} g_{\mu \nu} R=\epsilon\left\{\phi_{; \mu} \phi_{; \nu}-\frac{2-a}{2} g_{\mu \nu} \phi^{; \rho} \phi_{; \rho}\right\}+(3-2 a) g_{\mu \nu} V(\phi), \\
& \square \phi+(a-1) \frac{\phi^{; \rho} \phi^{; \sigma} \phi_{; \rho ; \sigma}}{\phi_{; \alpha} \phi^{; \alpha}}=-\epsilon(3-2 a) V_{\phi},
\end{aligned}
$$

where $a$ is the free parameter of Rastall's theory, and the equations of GR are restored in the case $a=1 ; \epsilon= \pm 1$, so that $\epsilon=+1$ corresponds to a canonical scalar field, and $\epsilon=-1$ to a phantom one.

\footnotetext{
${ }^{3}$ The Rastall parameter $a$ is related to the other frequently used parameter $\lambda$ by $a=\frac{3 \lambda-2}{2 \lambda-1}$.
} 
Equations (11) can be rewritten as

$$
R_{\mu \nu}=\epsilon\left\{\phi_{; \mu} \phi_{; \nu}+\frac{1-a}{2} g_{\mu \nu} \phi^{; \rho} \phi_{; \rho}\right\}-(3-2 a) g_{\mu \nu} V(\phi),
$$

We are going to discuss the linear stability of static, spherically symmetric solutions of the theory under spherically symmetric perturbations. Accordingly, we consider the general spherically symmetric metric

$$
d s^{2}=e^{2 \gamma} d t^{2}-e^{2 \alpha} d u^{2}-e^{2 \beta} d \Omega^{2}
$$

where $\alpha, \beta, \gamma$ are functions of $u$ and $t$, and $d \Omega^{2}$ is the line element on a unit sphere. We also assume $\phi=\phi(u, t)$.

Assuming that our perturbed system only slightly deflects from a static background solution, let us write the field equations, neglecting all terms nonlinear with respect to time derivatives:

$$
\begin{aligned}
-e^{2(\alpha-\gamma)}(\ddot{\alpha}+2 \ddot{\beta})+\gamma^{\prime \prime}+\gamma^{\prime}\left(\gamma^{\prime}-\alpha^{\prime}+2 \beta^{\prime}\right)- & =-\frac{\epsilon}{2}(1-a) \phi^{\prime 2}-(3-2 a) e^{2 \alpha} V, \\
-e^{2(\alpha-\gamma)} \ddot{\alpha}+\gamma^{\prime \prime}+2 \beta^{\prime \prime}-\alpha^{\prime}\left(\gamma^{\prime}+2 \beta^{\prime}\right)+\gamma^{\prime 2}+2 \beta^{\prime 2} & =-\frac{\epsilon}{2}(3-a) \phi^{\prime 2}-(3-2 a) e^{2 \alpha} V, \\
-e^{2(\alpha-\gamma)} \ddot{\beta}+\beta^{\prime \prime}+\beta^{\prime}\left(\gamma^{\prime}-\alpha^{\prime}+2 \beta^{\prime}\right)-e^{2(\alpha-\beta)} & =-\frac{\epsilon}{2}(1-a) \phi^{\prime 2}-(3-2 a) e^{2 \alpha} V, \\
\dot{\beta}^{\prime}+\left(\beta^{\prime}-\gamma^{\prime}\right) \dot{\beta}-\beta^{\prime} \dot{\alpha} & =-\frac{\epsilon}{2} \phi^{\prime} \dot{\phi}, \\
a \phi^{\prime \prime}+\left[\gamma^{\prime}-a \alpha^{\prime}+2 \beta^{\prime}\right] \phi^{\prime}-e^{2(\alpha-\gamma)} \ddot{\phi} & =\epsilon(3-2 a) e^{2 \alpha} V_{\phi}
\end{aligned}
$$

(the overdot stands for $/ \mathrm{t}$, the prime for $/ \mathrm{u}$, the subscript $\phi$ for $d / d \phi$ ). For the static background we have the equations

$$
\begin{aligned}
\gamma^{\prime \prime}+\gamma^{\prime}\left(\gamma^{\prime}-\alpha^{\prime}+2 \beta^{\prime}\right) & =-\frac{\epsilon}{2}(1-a) \phi^{2}-e^{2 \alpha} W, \\
\gamma^{\prime \prime}+2 \beta^{\prime \prime}-\alpha^{\prime}\left(\gamma^{\prime}+2 \beta^{\prime}\right)+\gamma^{\prime 2}+2 \beta^{\prime 2} & =-\frac{\epsilon}{2}(3-a) \phi^{2}-e^{2 \alpha} W, \\
\beta^{\prime \prime}+\beta^{\prime}\left(\gamma^{\prime}-\alpha^{\prime}+2 \beta^{\prime}\right)-e^{2(\alpha-\beta)} & =-\frac{\epsilon}{2}(1-a) \phi^{\prime 2}-e^{2 \alpha} W, \\
a \phi^{\prime \prime}+\left[\gamma^{\prime}-a \alpha^{\prime}+2 \beta^{\prime}\right] \phi^{\prime} & =\epsilon e^{2 \alpha} W_{\phi},
\end{aligned}
$$

where $W(\phi)=(3-2 a) V(\phi)$. Assuming, with some small parameter $\varepsilon$,

$$
\phi(u, t)=\phi(u)+\delta \phi(u, t), \quad \delta \phi \sim \varepsilon \ll 1,
$$

and similarly for all other variables, we can write the perturbation equations in the linear order 


$$
\begin{aligned}
& O(\varepsilon): \\
& \begin{array}{r}
-e^{2(\alpha-\gamma)}(\delta \ddot{\alpha}+2 \ddot{\delta} \beta)+\delta \gamma^{\prime \prime}+ \\
\quad \delta \gamma^{\prime}\left(\gamma^{\prime}-\alpha^{\prime}+2 \beta^{\prime}\right)+\gamma^{\prime}\left(\delta \gamma^{\prime}-\delta \alpha^{\prime}+2 \delta \beta^{\prime}\right) \\
=-\epsilon(1-a) \phi^{\prime} \delta \phi^{\prime}-e^{2 \alpha}\left(2 \delta \alpha W+W_{\phi} \delta \phi\right), \\
-e^{2(\alpha-\gamma)} \delta \ddot{\alpha}+\delta \gamma^{\prime \prime}+2 \delta \beta^{\prime \prime}-\delta \alpha^{\prime}\left(\gamma^{\prime}+2 \beta^{\prime}\right)-\alpha^{\prime}\left(\delta \gamma^{\prime}+2 \delta \beta^{\prime}\right)+2 \delta \gamma \gamma^{\prime}+4 \beta^{\prime} \delta \beta^{\prime} \\
=-\epsilon(3-a) \phi^{\prime} \delta \phi^{\prime}-e^{2 \alpha}\left(2 \delta \alpha W+W_{\phi} \delta \phi\right), \\
-e^{2(\alpha-\gamma)} \delta \ddot{\beta}+\delta \beta^{\prime \prime}+\delta \beta^{\prime}\left(\gamma^{\prime}-\alpha^{\prime}+2 \beta^{\prime}\right)+\beta^{\prime}\left(\delta \gamma^{\prime}-\delta \alpha^{\prime}+2 \delta \beta^{\prime}\right)-2 e^{2(\alpha-\beta)}(\delta \alpha-\delta \beta) \\
=-\epsilon(1-a) \phi^{\prime} \delta \phi^{\prime}-e^{2 \alpha}\left(2 \delta \alpha W+W_{\phi} \delta \phi\right), \\
\delta \dot{\beta}^{\prime}+\left(\beta^{\prime}-\gamma^{\prime}\right) \delta \dot{\beta}-\beta^{\prime} \delta \dot{\alpha}=-\frac{\epsilon}{2} \phi^{\prime} \delta \dot{\phi}, \\
-e^{2(\alpha-\gamma)} \delta \ddot{\phi}+a \delta \phi^{\prime \prime}+\left[\gamma^{\prime}-a \alpha^{\prime}+2 \beta^{\prime}\right] \delta \phi^{\prime}+\left[\delta \gamma^{\prime}-a \delta \alpha^{\prime}+2 \delta \beta^{\prime}\right] \phi^{\prime} \\
=\epsilon e^{2 \alpha}\left(2 \delta \alpha W_{\phi}+W_{\phi \phi} \delta \phi\right) .
\end{array}
\end{aligned}
$$

These equations are written in the most genearl form and contain two kinds of arbitrariness: the choice of the radial coordinate $u$ in the background static metric and the perturbation gauge that fixes the reference frame in perturbed space-time.

\section{Master equation and a discrepancy}

As in GR, this system possesses only one dynamic degree of freedom connected with the scalar perturbation $\delta \phi$. Accordingly, the perturbation equations can be used to exclude the metric perturbations and to obtain a single "master equation" for $\delta \phi$. This can be achieved most conveniently using the gauge $\delta \beta \equiv 0$. The perturbation equations become

$$
\begin{aligned}
& -e^{2(\alpha-\gamma)} \delta \ddot{\alpha}+\delta \gamma^{\prime \prime}+\delta \gamma^{\prime}\left(2 \gamma^{\prime}-\alpha^{\prime}+2 \beta^{\prime}\right)-\gamma^{\prime} \delta \alpha^{\prime}=-\epsilon(1-a) \phi^{\prime} \delta \phi^{\prime}-e^{2 \alpha}\left(2 W \delta \alpha+W_{\phi} \delta \phi\right), \\
& -e^{2(\alpha-\gamma)} \delta \ddot{\alpha}+\delta \gamma^{\prime \prime}-\delta \alpha^{\prime}\left(\gamma^{\prime}+2 \beta^{\prime}\right)-\left(\alpha^{\prime}-2 \gamma^{\prime}\right) \delta \gamma^{\prime}=-\epsilon(3-a) \phi^{\prime} \delta \phi^{\prime}-e^{2 \alpha}\left(2 W \delta \alpha+W_{\phi} \delta \phi\right), \\
& \beta^{\prime}\left(\delta \gamma^{\prime}-\delta \alpha^{\prime}\right)-2 e^{2(\alpha-\beta)} \delta \alpha=-\epsilon(1-a) \phi^{\prime} \delta \phi^{\prime}-e^{2 \alpha}\left(2 W \delta \alpha+W_{\phi} \delta \phi\right), \\
& -\beta^{\prime} \delta \dot{\alpha}=-\frac{\epsilon}{2} \phi^{\prime} \delta \dot{\phi} \\
& a \delta \phi^{\prime \prime}+\left[\gamma^{\prime}-a \alpha^{\prime}+2 \beta^{\prime}\right] \delta \phi^{\prime}+\left[\delta \gamma^{\prime}-a \delta \alpha^{\prime}\right] \phi^{\prime}-e^{2(\alpha-\gamma)} \delta \ddot{\phi}=\epsilon e^{2 \alpha}\left(2 W_{\phi} \delta \alpha+W_{\phi \phi} \delta \phi\right) .
\end{aligned}
$$

Equation (22) is easily integrated in $t$ leading to

$$
\delta \alpha=\eta(u) \delta \phi+\xi(u)
$$

where $\xi(u)$ is an arbitrary function corresponding to possible static perturbations while $\eta(u)$ is defined as

$$
\eta=\epsilon \phi^{\prime} /\left(2 \beta^{\prime}\right)
$$

On the other hand, the difference of (19) and (20) ) gives

$$
\delta \gamma^{\prime}=\eta \delta \phi^{\prime}-\eta^{\prime} \delta \phi .
$$


Ignoring static perturbations (that is, putting $\xi(u) \equiv 0)$, a substitution of (24) and (26) into (23) leads to the final master equation

$$
\begin{aligned}
-e^{2(\alpha-\gamma)} \delta \ddot{\phi}+a \delta \phi^{\prime \prime}+\left[2 \beta^{\prime}+\right. & \left.\gamma^{\prime}-a \alpha^{\prime}+\eta(1-a) \phi^{\prime}\right] \delta \phi^{\prime} \\
& +\left[-(1+a) \eta^{\prime} \phi^{\prime}-\epsilon e^{2 \alpha}\left(2 \eta W_{\phi}+W_{\phi \phi}\right)\right] \delta \phi=0, .
\end{aligned}
$$

whose analysis for particular solutions of the background equations should lead to definite conclusions on their stability or instability.

However, $\delta \gamma^{\prime}$ may be alternatively calculated from (21), which gives

$$
2 \beta^{\prime} \delta \gamma^{\prime}=\epsilon a \phi^{\prime} \delta \phi^{\prime}+e^{2 \alpha}\left[2\left(e^{-2 \beta}-W\right) \delta \alpha-W_{\phi} \delta \phi\right] .
$$

Comparing (26) and (28) and using the background equations, we obtain the relation

$$
(1-a) \phi^{\prime} \delta \phi^{\prime}=(1-a)\left[\phi^{\prime \prime}-\alpha^{\prime} \phi^{\prime}+\eta \phi^{\prime 2}\right] \delta \phi
$$

(the calculation is most conveniently carried out using the harmonic radial coordinate, such that $\alpha=2 \beta+\gamma)$. We immediately see that at $a=1$, that is, in GR, this equation becomes an identity, thus confirming that the stability study in GR remains consistent. However, with $a \neq 1$, in Rastall's theory, Eq. (29) is nontrivial, and its integration in $u$ gives

$$
\delta \phi=F(t) \phi^{\prime} e^{\alpha+Q(u)}, \quad Q(u)=\int \eta(u) \phi^{\prime}(u) d u
$$

where $F(t)$ is an arbitrary function of time, which only should be small, i.e., $O(\varepsilon)$.

Now, we can substitute (30) to the master equation (27) and observe the usual separation of

variables: the quantity $\ddot{F} / F$ will be equal to a certain combination of functions taking part in the background solution, hence this combination is equal to some separation constant. Such a condition, is, in general, not satisfied by the background solution, which makes the whole stability study inconsistent.

We will confirm this observation using two known background solutions of Rastall theory as examples. Using one of them, we will demonstrate that invoking a nonzero $\xi(u)$ in (24) does not solve the consistency problem.

\section{Special solutions}

\section{1 $a=-1, V=0$}

In this case, the solution has been obtained using the quasiglobal radial coordinate such that $\alpha=-\gamma$, and the background equation are

$$
\begin{aligned}
\gamma^{\prime \prime}+2 \gamma^{\prime}\left(\gamma^{\prime}+\beta^{\prime}\right) & =-\epsilon \phi^{2}, \\
\gamma^{\prime \prime}+2 \beta^{\prime \prime}+2 \gamma^{\prime}\left(\gamma^{\prime}+\beta^{\prime}\right)+2 \beta^{\prime 2} & =-2 \epsilon \phi^{2}, \\
\beta^{\prime \prime}+2 \beta^{\prime}\left(\gamma^{\prime}+\beta^{\prime}\right)-e^{-2(\gamma+\beta)} & =-\epsilon \phi^{2}, \\
-\phi^{\prime \prime}+2 \beta^{\prime} \phi^{\prime} & =0 .
\end{aligned}
$$


The background solutions are possible only for $\epsilon=-1$ and are given by (denoting now the radial coordinates by $x$ )

$$
\begin{aligned}
& d s^{2}=A(x) d t^{2}-\frac{d x^{2}}{A(x)}-\sqrt{\frac{3}{2 C^{2}}} \frac{d \Omega^{2}}{x}, \\
& A(x)=K / x-(C / \sqrt{6}) x^{3}, \\
& \phi=\sqrt{3 / 2} \ln x+\phi_{0},
\end{aligned}
$$

where $\phi_{0}, C$ and $K$ are integration constants. The metric has the same properties as in the $\mathrm{k}$ essence case studied in Ref. [6], with a horizon at $x=\left(\frac{\sqrt{6} K}{C}\right)^{1 / 4}$ and a singular spatial infinity $x \rightarrow \infty$. Unlike [6], however, the scalar field is here defined as to span from minus to plus infinity.

The master equation (27) now reduces to

$$
e^{-4 \gamma} \delta \ddot{\phi}+\delta \phi^{\prime \prime}+\frac{2}{x} \delta \phi^{\prime}=0
$$

Two different expressions for $\delta \gamma^{\prime}$, obtained as described above, are

$$
\delta \gamma^{\prime}=\sqrt{3 / 2} \delta \phi^{\prime}=-\sqrt{3 / 2} \delta \phi^{\prime}-\sqrt{6} e^{2 \alpha-2 \beta} \delta \phi .
$$

Substituting the background solution, we are able to integrate the last equality in $x$, obtaining

$$
\delta \phi=F(t) \psi(x), \quad \psi(x)=\left(K-(C / \sqrt{6}) x^{4}\right)^{-1 / 2} .
$$

Substituting (401) into (38) and separating the variables, we obtain

$$
-\frac{\ddot{F}}{F}=\frac{\psi^{\prime \prime}}{\psi}+\frac{2}{x} \frac{\psi^{\prime}}{\psi}=\text { const. }
$$

It is easy to verify that the last equation does not hold for $\psi(x)$ given by (40)).

\section{2 $a=0, V=\Lambda=$ const}

We are using here the the harmonic radial coordinate, such that $\alpha=2 \beta+\gamma$. The background equations are

$$
\begin{aligned}
\gamma^{\prime \prime} & =-\frac{\epsilon}{2} \phi^{2}-3 e^{2 \alpha} \Lambda, \\
\gamma^{\prime \prime}+2 \beta^{\prime \prime}-\alpha^{\prime 2}+\gamma^{\prime 2}+2 \beta^{\prime 2} & =-\frac{3}{2} \epsilon \phi^{2}-3 e^{2 \alpha} \Lambda, \\
\beta^{\prime \prime}-e^{2(\gamma+\beta)} & =-\frac{\epsilon}{2} \phi^{2}-3 e^{2 \alpha} \Lambda, \\
\alpha^{\prime} \phi^{\prime} & =0 .
\end{aligned}
$$

The last equation implies $\alpha=$ const, which can be rescaled to $\alpha=0$ by a trivial redefinition of the radial coordinate. The solution has the form

$$
\begin{aligned}
& d s^{2}=\frac{9 b^{4}}{\cosh ^{4} b u} d t^{2}-d u^{2}-\frac{\cosh ^{2} b u}{3 b^{2}} d \Omega^{2}, \\
& \phi^{\prime}= \pm b \sqrt{6-\frac{4}{\cosh ^{2} b u}}, \quad \epsilon=-1, \quad b=\sqrt{\Lambda} .
\end{aligned}
$$


Equation (231) for scalar field perturbations now reads

$$
e^{-2 \gamma} \delta \ddot{\phi}-\phi^{\prime} \delta \gamma^{\prime}=0
$$

Two alternative expressions for $\delta \gamma^{\prime}$ are (26) and, as given by (28) after using the background solution,

$$
\delta \gamma^{\prime}=\frac{3}{2} \phi^{\prime} \delta \phi .
$$

Comparing (26) and (49), we obtain

$$
\eta \delta \phi^{\prime}-\eta^{\prime} \delta \phi=\frac{3}{2} \phi^{\prime} \delta \phi .
$$

We can integrate this equation, finding the spatial behavior of $\delta \phi$ :

$$
\delta \phi=F(t) \frac{\phi^{\prime}}{\beta^{\prime}} e^{-3 \beta}, \quad F(t)=\text { arbitrary function. }
$$

Substituting it to the master equation (48) with $\delta \gamma^{\prime}$ given by (49), we arrive at

$$
\frac{2}{3} \frac{\ddot{F}}{F}=\phi^{\prime 2} e^{-4 \beta}=\text { const. }
$$

The latter equality does not hold for our background solution.

We can ask if we can cure this problem by considering the "integration constant" $\xi(u)$ that appears in (24). Then the two expressions for $\delta \gamma^{\prime}$ become

$$
\delta \gamma^{\prime}=\eta \delta \phi^{\prime}-\eta^{\prime} \delta \phi-\xi^{\prime}=\frac{3}{2} \phi^{\prime} \delta \phi-3 \beta^{\prime} \xi .
$$

This relation is integrated giving

$$
\delta \phi=\eta e^{-3 \beta}[F(t)+H(u)], \quad H(u)=\int \frac{e^{3 \beta}}{\eta^{2}}\left(\xi^{\prime}-3 \beta^{\prime} \xi\right) d u,
$$

where $H(u)$ is actually an arbitrary function of $u$ due to arbitrariness of $\xi$. Inserting this $\delta \phi$ to the master equation, we obtain

$$
\ddot{F}-\frac{3}{2} \phi^{\prime 2} e^{-4 \beta}[F(t)+H(u)]-6 \beta^{\prime 2} e^{-\beta} \xi=0 .
$$

Differentiating (55) with respect to $u$, we obtain a relation between functions of $u$ (making it possible to calculate $\xi(u)$ in terms of the background functions) and also that $F=$ const. As could be expected, the assumption $\xi(u) \neq 0$ leads to a purely static perturbation of the background, and time-dependent perturbations turn out to be impossible. Thus invoking $\xi(u)$ only produces a static perturbation but does not solve the consistency problem for time-dependent perturbations. 


\section{Conclusion}

We have performed a stability analysis of the exact black hole-type solutions found in the context of the Rastall theory of gravity in the presence of a self-interacting scalar field, which were originally reported in Ref. [5]. The corresponding metrics are the same as those found in the context of k-essence theories [6]. This coincidence of the metrics found in so different contexts (but with quite different behaviors of the corresponding scalar fields) was discussed in detail in Ref. [7]. The perturbation analysis of the k-essence solutions was carried out in [8, and it was concluded that they are unstable. Hence, it was natural to perform a similar analysis for the corresponding Rastall solutions since the equivalence with the k-essence solutions may not be preserved at the perturbative level.

A surprising results is that such a stability analysis in the Rastall case is inconsistent: linear time-dependent spherically symmetric perturbations simply do not exist. In this sense, the solutions may be said to be stable under such perturbations. The reason for our conclusion is that in the Rastall theory different combinations of the perturbed equations lead to different "master" equations for the scalar field perturbation $\delta \phi$. This problem does not exist for k-essence solutions. We cannot attribute this feature to a wrong choice of the coordinates or the perturbation gauge since, as discussed, e.g., in [10 12], the perturbation method employed here is equivalent to a gauge-invariant method.

Very probably the roots of the inconsistency detected for Rastall black hole-type solutions come from the absence of a Lagrangian formulation of this theory. It must be remarked that a similar inconsistency was found in the cosmological context, also with a scalar field as a matter source. But, for the cosmological solutions the inconsistency has been cured by introducing ordinary baryonic matter. In the static, spherically symmetric case studied in [5] such an extension is less obvious.

There have been some attempts to find a Lagrangian formulation for the Rastall theory, see, e.g.,. [13,14], but the resulting theories were not completely equivalent to the Rastall one, or requires a completely new geometric framework: using these formulations, we, strictly speaking, go away from the original context of the Rastall theory.

The results reported here may point out at some restrictions inherent to the applicability of the Rastall theory, and even maybe of any non-Lagrangian theory. We hope to extend the present analysis in order to try to answer this question in our future works.

\section{Acknowledgments:}

J.C. Fabris and O.F. Piattella thank CNPq (Brazil) and FAPES (Brazil) for partial financial support. D.C. Rodrigues was financed in part by the Coordenação de Aperfeitoamento de Pessoal de Nível Superior - Brasil (CAPES) - Finance Code 001. E.C.O. Santos thanks FAPES (Brazil) for financial support. The work of K. Bronnikov was supported by the RUDN University program 5-100 and by the Russian Foundation for Basic Research Project 19-02-00346. The work of K.B. was also performed within the framework of the Center FRPP supported by MEPhI Academic Excellence Project (contract No. 02.a03.21.0005, 27.08.2013).

\section{References}

[1] P. Rastall, Phys. Rev. D6, 3357(1972).

[2] G.F. Silva, O.F. Piattella, J.C. Fabris, L. Casarini and T.O. Barbosa, Grav. Cosmol. 19, 156 (2013). 
[3] C.E.M. Batista, M.H. Daouda, J.C. Fabris, O.F. Piattella and Davi C. Rodrigues, Phys. Rev. D85, 084008 (2012).

[4] C. Gao, M. Kunz, A. R. Liddle and D. Parkinson, Phys. Rev. D81, 043520 (2010).

[5] K.A. Bronnikov, J.C. Fabris, O.F. Piattella and E. Santos, Gen. Rel. Grav. 48, 162 (2016).

[6] K.A. Bronnikov, J.C. Fabris and Denis C. Rodrigues, Grav. Cosmol. 22, 26 (2016).

[7] K.A. Bronnikov, J.C. Fabris, O.F. Piattella, Denis C. Rodrigues and E.C. Santos, Eur. Phys. J. C 77, 409 (2017).

[8] K.A. Bronnikov, J.C. Fabris and Denis C. Rodrigues, Int. J. Mod. Phys. D 29, 2050016 (2020).

[9] J.C. Fabris, M.H. Daouda and O.F. Piattella, Phys. Lett. B711, 232(2012).

[10] J.A. Gonzalez, F.S. Guzman and O. Sarbach, Class. Quantum Grav. 26, 015010 (2009); arXiv: 0806.0608.

[11] K.A. Bronnikov, J.C. Fabris and A. Zhidenko, Eur. Phys J. C 71, 1791 (2011); arXiv: 1109.6576.

[12] K.A. Bronnikov, Particles 2018, 1, 5 (2018); arXiv: 1802.00098.

[13] L. Smalley, Il Nuovo Cimento B80, 42(1984).

[14] R.V. dos Santos and J.A.C. Nogales, Cosmology from a Lagrangian formulation for Rastall's theory, arXiv: 1701.08203. 